\title{
Gleason-Type Derivations of the Quantum Probability Rule for Generalized Measurements
}

\author{
Carlton M. Caves, ${ }^{1}$ Christopher A. Fuchs, ${ }^{2, *}$ Kiran Manne, ${ }^{1}$ and Joseph M. Renes ${ }^{1}$ \\ ${ }^{1}$ Department of Physics and Astronomy, \\ University of New Mexico, \\ Albuquerque, New Mexico 87131-1156, USA \\ ${ }^{2}$ Quantum Information and Optics Research, \\ Bell Labs, Lucent Technologies, \\ 600-700 Mountain Avenue, \\ Murray Hill, New Jersey 07974, USA
}

(Dated: 2003 June 26)

\begin{abstract}
We prove a Gleason-type theorem for the quantum probability rule using frame functions defined on positive-operator-valued measures (POVMs), as opposed to the restricted class of orthogonal projection-valued measures used in the original theorem. The advantage of this method is that it works for two-dimensional quantum systems (qubits) and even for vector spaces over rational fieldssettings where the standard theorem fails. Furthermore, unlike the method necessary for proving the original result, the present one is rather elementary. In the case of a qubit, we investigate similar results for frame functions defined upon various restricted classes of POVMs. For the so-called trine measurements, the standard quantum probability rule is again recovered.
\end{abstract}

\section{INTRODUCTION}

Two theorems position themselves at the foundation of quantum theory: the Kochen-Specker theorem [1] and Gleason's theorem [2]. The former rules out the possibility of predicting experimental outcomes with certainty, and - failing this certainty - the latter prescribes the permitted probabilistic descriptions. Both are pivotal to an understanding of quantum mechanics that begins with primitive notions about measurement - the subject of this paper.

To adopt this perspective, imagine that for any experiment, there exist mathematical objects which represent the possible outcomes. Likewise, there exists a mathematical object the experimenter uses to describe the system under investigation. The minimal task of any physical theory is to determine what these objects are and to use them to furnish the probabilities for the various outcomes.

Here the traditional viewpoint of physical theory is stood on its head. Usually the system and its propertieswhatever can be said about them - are regarded as fundamental. Physical systems are considered objectively, outside the context of measurements, real or potential. Within this traditional viewpoint the question for physics is this: What are the properties of physical systems, and what laws do they obey? Notoriously this leads to the quantum measurement problem. Having considered physical systems to obey quantum mechanics, what are we to make of measurements? How does measurement fit into the framework of quantum theory?

In this paper we view measurements as fundamental, obviating the measurement problem. The main task is an operational one: How should measurements be described, and what predictions about their outcomes can be made? Properties of physical systems, e.g., position and momentum, are useful if they help in this task, but are ultimately secondary. Though the conventional measurement problem does not arise in this context, a new question emerges, just as daunting as the original: Why the Hilbert-space description of measurement outcomes [3, 4, 5]?

Leaving this question aside as too ambitious for the present, we start by assuming the form of measurements as given by classical or quantum theory. In classical physics, the measurement objects are the points in a phase space, while in quantum physics, they are traditionally one-dimensional projectors on a Hilbert space. Classically only one measurement exists - a full accounting of the phase space. In quantum mechanics, on the other hand, any complete set of one-dimensional orthogonal projectors suffices.

The description of the system can be given, in classical mechanics, by a phase-space point. This point is the "true" point - others are "false" - so the outcome of a measurement can be predicted with certainty. Attempting such a concrete description in quantum mechanics is ruled out by the Kochen-Specker theorem: There is no way to assign truth and falsity to all the one-dimensional projectors in such a way that in any measurement there is only one true outcome. At this point in the development, quantum mechanics becomes an irreducibly probabilistic theory; the possibility of underlying certainties has been ruled out.

\footnotetext{
* Temporary Address: Communication Networks Research Institute, Dublin Institute of Technology, Rathmines Road, Dublin 6, Ireland.
} 
With certainties ruled out, Gleason's theorem delineates the allowable descriptions of the system, i.e., the form the probabilities can take. Keeping with the linear structure, every outcome probability is an inner product of the corresponding measurement projector and a density operator for the system. The density operator - any convex combination of one-dimensional projection operators - represents the description or "state" of the system. Thus Gleason's theorem gives a means to go from the structure of measurements to the structure of states. It immediately implies the Kochen-Specker result, as there are no density operators that yield probability distributions for all measurements that are valued only on zero and one.

Neither theorem holds for two-dimensional quantum systems, so-called qubits, as long as the measurement objects are restricted to being one-dimensional projection operators. We can include this outstanding case, however, by widening the class of allowed measurements to include positive-operator-valued measures (POVMs), comprised of measurement operators called effects, and in so doing, we also considerably simplify the derivation. This generalization is the subject of this paper. Section [1] describes how measurement outcomes in quantum mechanics are associated with effects. Section III shows that given the structure of effects, the usual quantum-mechanical probability rule follows simply, even for qubits. Section $\amalg$ investigates several specific restricted classes of measurements for qubits and what kinds of probability distributions these classes permit.

It should be emphasized from the outset that this is an inherently noncontextual approach, meaning that a shared outcome of two distinct measurements has the same probability in both contexts. This fact is not a consequence of this approach, but rather an assumption used in its construction. In this construction a description of the measurement device and a description of the physical system are composed into a probability for each possible outcome. In defining measurements by their physical set-ups, this approach initially pertains to each measurement situation individually. The Hilbert-space formalism we are using to describe measurements takes a further step by associating the same measurement object with outcomes in different measurements. For this reason, we assume that these outcomes have the same probabilities, this being the noncontextual assumption. To abandon this assumption at the level of finding allowed probability assignments via Gleason's theorem would be to ignore, wholly or partially, the framework provided by the vector-space structure of measurements. There are other, perhaps stronger arguments for noncontextuality [3] but the argument here is a minimal one that all the authors of this paper are willing to accept.

\section{EFFECT OPERATORS}

In the typical von Neumann formulation of quantum measurement theory, measurements are described by complete sets of orthogonal projection operators. Here we consider quantum measurements in their full generality, the so-called POVMs [6, 7]. A POVM is also a complete set of operators resolving the identity operator, but comprised of positive operators less than the identity. These operators, called effects, can also be characterized as Hermitian operators having eigenvalues in the unit interval. The set of effects in $d$ dimensions we denote by $\mathcal{E}_{d}$.

That $\mathcal{E}_{d}$ is a convex set is clear. The projection operators of all ranks, including the zero operator $\mathbf{0}$ and the identity operator $\mathbf{1}$, form the extreme points of $\mathcal{E}_{d}$, a fact that we demonstrate via the following construction. For a given effect $E$, order its $d$ eigenvalues (including zero eigenvalues) from smallest to largest, $\left\{\lambda_{1}, \ldots, \lambda_{d}\right\}$. Associated with each eigenvalue $\lambda_{j}$ is a one-dimensional projector $\pi_{j}$ onto the corresponding eigenvector; these projectors can be chosen to make up a complete, orthonormal set. Now form the projection operators $\Pi_{k}=\sum_{j=k}^{d} \pi_{j}$. Clearly $\Pi_{1}=1$ and $\Pi_{d}=\pi_{d}$. These projectors get smaller in rank as the index gets bigger. The effect $E$ can be written as the following convex combination:

$$
E=\lambda_{1} \Pi_{1}+\sum_{m=2}^{d}\left(\lambda_{m}-\lambda_{m-1}\right) \Pi_{m}+\left(1-\lambda_{d}\right) \mathbf{0} .
$$

The zero operator is included to make the sum of the coefficients unity without affecting $E$.

Since every effect can be expanded as a convex combination of projectors, only projectors can be extreme points of $\mathcal{E}_{d}$. To show that all the projectors are extreme points, we need to show that a projector $\Pi$ cannot be written as a proper convex combination of other projectors, i.e., cannot be written as $\Pi=a \Pi_{1}+(1-a) \Pi_{2}$, where $0<a<1$ and $\Pi_{1} \neq \Pi_{2}$. To show this, suppose $\Pi$ could be so written. For any normalized vector $|\psi\rangle$, we have $\langle\psi|\Pi| \psi\rangle=$ $a\left\langle\psi\left|\Pi_{1}\right| \psi\right\rangle+(1-a)\left\langle\psi\left|\Pi_{2}\right| \psi\right\rangle$. If $|\psi\rangle$ is in the null subspace of $\Pi$, i.e., is orthogonal to the support of $\Pi$, we have $\langle\psi|\Pi| \psi\rangle=0$, which implies that $\left\langle\psi\left|\Pi_{1}\right| \psi\right\rangle=\left\langle\psi\left|\Pi_{2}\right| \psi\right\rangle=0$; this shows that the supports of $\Pi_{1}$ and $\Pi_{2}$ are contained in the support of $\Pi$. If $|\psi\rangle$ is in the support of $\Pi$, we have $\langle\psi|\Pi| \psi\rangle=1$, which implies that $\left\langle\psi\left|\Pi_{1}\right| \psi\right\rangle=\left\langle\psi\left|\Pi_{2}\right| \psi\right\rangle=1$; this shows that the support of $\Pi$ is contained in the supports of $\Pi_{1}$ and $\Pi_{2}$. Together these conclusions imply that $\Pi_{1}=\Pi_{2}=\Pi$, contradicting our assumption of a proper convex combination for $\Pi$. We conclude that the extreme points of $\mathcal{E}_{d}$ are the projectors of all ranks, including $\mathbf{0}$. 
Projection operators are a limiting case of effect operators, the latter being a "fuzzy" or "unsharp" version of the former by convex combination. Similarly, the von Neumann projective measurements are a limiting case of POVMs.

In two dimensions the set of effects, $\mathcal{E}_{2}$, has an appealing geometric picture. Beginning with the parameterization of Hermitian operators by the Pauli matrices, we write the general two-dimensional effect as

$$
E=r \mathbf{1}+\vec{s} \cdot \vec{\sigma}=r \mathbf{1}+s \hat{n} \cdot \vec{\sigma}
$$

where $\hat{n}$ is a unit vector. The eigenvalues $r \pm\|\vec{s}\|=r \pm s$ must lie in the unit interval, which is equivalent to the conditions $0 \leq r \leq 1,0 \leq s \leq r$, and $0 \leq s \leq 1-r$. Since $s$ characterizes the radius of a sphere, the full set can be pictured in the following way: Starting with the unit interval for $r$, associate with each point a ball of radius $r$ for $r \leq 1 / 2$ and of radius $1-r$ for $r>1 / 2$. The set of two-dimensional effects is thus the intersection of two three-dimensional cones, both having an opening angle of $45^{\circ}$, one extending up from a vertex at $r=s=0(E=\mathbf{0})$ and the other extending down from a vertex at $r=1, s=0(E=1)$. The intersection of the boundaries of the two cones, $r=s=1 / 2$, is the surface of the Bloch sphere, where the effects are one-dimensional projectors. The effects on the boundary of the lower cone, $r=s \leq 1 / 2$, are multiples of one-dimensional projectors.

\section{THE QUANTUM PROBABILITY RULE}

The task of quantum theory is, minimally, to associate with every measurement a probability distribution for its outcomes. We assume this is done noncontextually for the reasons given above. The probability rule is thus a function from the set of effects to the unit interval, which is normalized on any subset that makes up a POVM. Such a function is known as a frame function. More precisely, a frame function is a function $f: \mathcal{E}_{d} \rightarrow[0,1]$ that satisfies

$$
\sum_{E_{j} \in X} f\left(E_{j}\right)=1
$$

on any subset $X=\left\{E_{j} \in \mathcal{E}_{d} \mid \sum_{j} E_{j}=\mathbf{1}\right\}$. This section is devoted to proving the following Gleason-type theorem, which was first proved by Busch [8].

Theorem 1 For every frame function $f: \mathcal{E}_{d} \rightarrow[0,1]$, there is a unique unit-trace positive operator $W$ such that $f(E)=(W, E)=\operatorname{tr}(W E)$.

The operator $W$ is the density operator that gives rise to the frame function probabilities $f(E)$. We divide the proof of this theorem into several parts, each of which occupies a subsection.

\section{A. Linearity with respect to the nonnegative rationals}

Every frame function is trivially additive, for consider two POVMs, $\left\{E_{1}, E_{2}, E_{3}\right\}$ and $\left\{E_{1}+E_{2}, E_{3}\right\}$. Clearly both are POVMs if either is, and the frame-function requirement immediately yields

$$
f\left(E_{1}\right)+f\left(E_{2}\right)=f\left(E_{1}+E_{2}\right) .
$$

¿From this we obtain a homogeneity property for multiplication by rational numbers. We can break an effect $n E$ into $m$ pieces to form the effect $(n / m) E$. Using the additivity property twice, we obtain

$$
m f\left(\frac{n}{m} E\right)=f(n E)=n f(E) \quad \Longrightarrow \quad f\left(\frac{n}{m} E\right)=\frac{n}{m} f(E) \text {. }
$$

The function $f$ is thus established to be linear in the nonnegative rationals. We can extend to full linearity by proving continuity. Alternately, adopting the strategy of Busch [8], we can demonstrate the homogeneity of $f$, from which linearity follows immediately. These two arguments are taken up in turn in the next two subsections.

\section{B. Continuity}

Continuity of the frame function can be established via reductio ad absurdum: A contradiction with the definition of a frame function arises if $f$ is discontinuous. Recall the definition of continuity for metric spaces: $f$ is continuous 
at $x_{0}$ if for all $\epsilon>0$, there exists a $\delta>0$ such that $\left|f(x)-f\left(x_{0}\right)\right|<\epsilon$ for all $x$ satisfying $\left|x-x_{0}\right|<\delta$. On the space of operators, we use the Hilbert-Schmidt inner product $(A, B)=\operatorname{tr}\left(A^{\dagger} B\right)$ and the associated norm $|A| \equiv \sqrt{(A, A)}$.

Consider first continuity at the zero operator [since $f(E)=f(E+\mathbf{0})=f(E)+f(\mathbf{0})$, we know that $f(\mathbf{0})=0$ ]. If we assume $f$ is discontinuous at the zero operator, then there exists an $\epsilon>0$ such that for all $\delta>0$, there exists an effect $E$ satisfying $|E|<\delta$ and $f(E) \geq \epsilon$. Choose $\delta=1 / N<\epsilon$, where $N$ is an integer, and let $E$ be an effect satisfying $|E|<1 / N$ and $f(E) \geq \epsilon$. Now $F=N E$ satisfies $|F|=N|E|<1$, which implies that $F$ is an effect, since the sum of the squares of its eigenvalues is less than 1. But we also have from additivity that $f(F)=N f(E) \geq N \epsilon>1$, contradicting the definition of a frame function. We conclude that $f$ is continuous at the zero operator.

We can easily translate the continuity at $\mathbf{0}$ to the entire set of effects. To prove continuity at an arbitrary effect $E_{0}$, we need to consider neighboring operators $E$ and the difference $E-E_{0}$. Diagonalizing the difference, we can write $E-E_{0}=A-B$, where $A$ is the nonnegative-eigenvalue part of the eigendecomposition and $-B$ is the negativeeigenvalue part. It is clear that $A$ and $B$ are positive operators satisfying $|A|,|B| \leq|A-B|=\left|E-E_{0}\right|$, which implies that $A$ and $B$ are effects (provided $\left|E-E_{0}\right| \leq 1$ ). Applying the frame function to the equation $E+B=E_{0}+A$ yields $f(E)-f\left(E_{0}\right)=f(A)-f(B)$ by additivity. Invoking continuity at zero establishes that for every $\epsilon=\epsilon^{\prime} / 2>0$, there exists a $\delta>0$ such that $|A|,|B|<\delta \Rightarrow f(A), f(B)<\epsilon^{\prime}$. Thus if $\left|E-E_{0}\right|=|A-B|<\delta$, we have $|A|,|B|<\delta$ and $\left|f(E)-f\left(E_{0}\right)\right|=|f(A)-f(B)| \leq|f(A)|+|f(B)|<2 \epsilon^{\prime}=\epsilon$. This establishes the continuity of $f$ on all of $\mathcal{E}_{d}$, which in turn shows that $f$ is a linear function on $\mathcal{E}_{d}$.

\section{Homogeneity}

An alternative route to linearity is to prove the homogeneity of the frame function. Following Busch's proof, we first note that the frame function preserves order; i.e., if $E_{1}<E_{2}$ for any pair of measurement operators, then $f\left(E_{1}\right) \leq f\left(E_{2}\right)$. This follows immediately from the definition, for $E_{1}<E_{2} \Leftrightarrow E_{2}-E_{1} \equiv E_{3}>0$, so $E_{3}$ is an effect. Writing $E_{2}=E_{1}+E_{3}$, which implies $f\left(E_{2}\right)=f\left(E_{1}\right)+f\left(E_{3}\right)$ by additivity, we find that $f\left(E_{1}\right) \leq f\left(E_{2}\right)$ since $f\left(E_{3}\right) \geq 0$.

Now the pinching theorem can be used to establish the homogeneity of $f$. Consider two sequences of rational numbers sharing the same irrational limit $\alpha:\left\{q_{i}\right\}$ is an increasing sequence and $\left\{p_{i}\right\}$ a decreasing sequence. By order preservation and linearity in the nonnegative rationals, we have

$$
q_{i} f(E)=f\left(q_{i} E\right) \leq f(\alpha E) \leq f\left(p_{i} E\right)=p_{i} f(E)
$$

for all $i$. The pinching theorem shows that $f(\alpha E)=\alpha f(E)$, establishing that $f$ is a homogeneous and, hence, linear function.

\section{Linearity and the inner product}

Since the frame function is linear, it arises from an inner product. To show this, we first extend the definition of $f$ to the entire vector space of operators; then it is a trivial theorem of linear algebra to recast a linear function on a vector space as an inner product.

We extend the definition of the function in the most straightforward fashion. Let $H$ be an arbitrary Hermitian operator. Every such operator can be written as the difference of two positive operators $G_{1}$ and $G_{2}$; one way to do so is simply to diagonalize $H$ and to let $G_{1}$ be the positive-eigenvalue part and $-G_{2}$ the negative-eigenvalue part. Further, for any positive operator $G$ we can find a positive number $\alpha$ such that $\alpha E=G$ for some effect $E$. Now we define $f(H)=f\left(G_{1}\right)-f\left(G_{2}\right)=\alpha_{1} f\left(E_{1}\right)-\alpha_{2} f\left(E_{2}\right)$. Though the unravelling of $H$ is not unique, the extension is. Suppose $H=\alpha_{1} E_{1}-\alpha_{2} E_{2}=\alpha_{3} E_{3}-\alpha_{4} E_{4}$, which implies $\alpha_{1} E_{1}+\alpha_{4} E_{4}=\alpha_{2} E_{2}+\alpha_{3} E_{3}$. Choose $\beta$ such that $\beta \geq \max \left\{\alpha_{j}\right\}$ so that we have

$$
\frac{\alpha_{1}}{\beta} E_{1}+\frac{\alpha_{4}}{\beta} E_{4}=\frac{\alpha_{2}}{\beta} E_{2}+\frac{\alpha_{3}}{\beta} E_{3} .
$$

Since every operator is now in the original domain of $f$, we can apply the frame function to find

$$
\alpha_{1} f\left(E_{1}\right)+\alpha_{4} f\left(E_{4}\right)=\alpha_{2} f\left(E_{2}\right)+\alpha_{3} f\left(E_{3}\right) .
$$

The extension being manifestly linear, we now have a linear function $f$ on the entire space of Hermitian operators. It can be extended to all operators by complexification.

To rewrite this linear function as an inner product, choose an orthonormal operator basis $\left\{\tau_{j}\right\}$, and write an arbitrary operator as $A=\sum_{j} \tau_{j}\left(\tau_{j}, A\right)$. Clearly we have $f(A)=\sum_{j} f\left(\tau_{j}\right)\left(\tau_{j}, A\right)$. Now define $W$ as the unique 
solution of the $d^{2}$ equations $f\left(\tau_{j}\right)=\left(W, \tau_{j}\right)$, so that the frame function is $f(A)=\sum_{j}\left(W, \tau_{j}\right)\left(\tau_{j}, A\right)=(W, A)$. The $d^{2}$ equations are, of course, nonsingular since $\left\{\tau_{j}\right\}$ is an orthonormal basis.

The nonnegativity and normalization of the frame function induce the density operator properties of $W$, i.e., positivity and unit trace. Given an arbitrary normalized vector $|\psi\rangle$, we have $0 \leq f(|\psi\rangle\langle\psi|)=\langle\psi|W| \psi\rangle$, showing that $W$ is positive. The condition of unit trace follows from normalization:

$$
\operatorname{tr} W=(W, \mathbf{1})=\left(W, \sum_{j} E_{j}\right)=\sum_{j}\left(W, E_{j}\right)=\sum_{j} f\left(E_{j}\right)=1 .
$$

The reader should note that if we omit the extension of linearity to real numbers (Secs. IIIB and IIIC), the arguments in Secs. IIIA and IIID demonstrate the quantum probability rule for vector spaces over rational fields. This result has implications for recent discussions in the literature about the possibility of describing the finite precision of real-world measurements via vector spaces over complex numbers with rational parts (see, in particular, Refs. 9, 10, 11, 12]). We do not dwell on these points, noting only that our result shows that rational POVMs cannot be assigned truth values, the only frame functions being those derived from density operators. In our view, given that POVMs are the preferred description of finite-precision measurements, that is all that needs to be said about finite-precision quantum measurements.

\section{FRAME FUNCTIONS FOR QUBITS}

The POVM version of Gleason's theorem works even for qubits, unlike the original Gleason theorem, which was based on measurements described by one-dimensional orthogonal projectors. We now turn our attention specifically to qubits and investigate whether several restricted sets of POVMs enforce the quantum probability rule. In particular, we show that the quantum probability rule is necessitated by a particular subset of POVM measurements called trines. Other measurements are studied to shed light on what kinds of measurements yield the quantum probability rule and why.

\section{A. General description of restricted sets of POVMs}

To start, recall that the two-dimensional effects are parameterized by four real parameters as in Eq. (2.2). In any of the restricted sets of measurements considered in this section, the allowed POVMS are made up of effects that are multiples of one-dimensional projectors, i.e., $r=s \leq 1 / 2$ and $E=r(1+\hat{n} \cdot \vec{\sigma})$, and all the effects have the same value of $r$. An allowed POVM is thus specified by a set of unit vectors that sum to the zero vector; if there are $N$ outcomes in the POVM, then $r=1 / N$. Finally, we assume that the allowed POVMs are rotationally invariant; i.e., they are obtained by applying all possible 3-dimensional rotations to any particular POVM in the allowed subset.

With these assumptions, we have the following structure. For $N$-outcome POVMs, the allowed effects have the form

$$
E=\frac{1}{N}(1+\hat{n} \cdot \vec{\sigma})
$$

where $\hat{n}$ can be any unit vector. Frame functions defined on this set, i.e.,

$$
f\left(\frac{1}{N}(\mathbf{1}+\hat{n} \cdot \vec{\sigma})\right) \equiv F(\hat{n})=\sum_{l=0}^{\infty} \sum_{m=-l}^{l} c_{l m} Y_{l m}(\hat{n}),
$$

are functions on the unit sphere and thus can be expanded in terms of spherical harmonics. In writing the sphericalharmonic expansion, we are assuming that $F$ is continuous on the unit sphere. (Please be careful to note that this extra continuity assumption was not made in the full-fledged Gleason-type theorem of the previous section.) Properties of the spherical harmonics that we need in the following are the following: (i) the separation into $\theta$ and $\phi$ (or $n_{z}$ and $n_{x}+i n_{y}$ ) dependencies,

$$
Y_{l m}(\hat{n})=Y_{l m}(\theta, \phi)=\sqrt{\frac{(2 l+1)}{4 \pi} \frac{(l-m) !}{(l+m) !}} P_{l}^{m}(\cos \theta) e^{i m \phi}=h_{l m}\left(n_{z}\right)\left(n_{x}+i n_{y}\right)^{m},
$$

where $P_{l}^{m}(x)$ is an associated Legendre function, and (ii) the changes under conjugation, reflection, and parity,

$$
Y_{l m}(\hat{n})=(-1)^{m} Y_{l,-m}^{*}(\hat{n})=(-1)^{l+m} Y_{l m}(\pi-\theta, \phi)=(-1)^{m} Y_{l m}(\theta, \phi+\pi)=(-1)^{l} Y_{l m}(-\hat{n}) .
$$


Particularly useful is the form of Eq. (4.3) for $m=l: Y_{l l}(\hat{n}) \propto\left(n_{x}+i n_{y}\right)^{l}$.

The sought-after quantum rule is

$$
F(\hat{n})=\operatorname{tr}(W E)=\frac{1}{N}(1+\hat{n} \cdot \vec{P}),
$$

where $\vec{P}=\operatorname{tr}(W \vec{\sigma})$ is any 3 -vector such that $\|\vec{P}\| \leq 1$. The quantum rule evidently contains only $l=0,1$ spherical harmonics, with $c_{00}=\sqrt{4 \pi} / N, c_{10}=\sqrt{4 \pi / 3} P_{z} / N$, and $c_{1, \pm 1}=\sqrt{2 \pi / 3}\left(\mp P_{x}+i P_{y}\right) / N$.

Now let the set of unit vectors $\left\{\hat{n}_{1}, \ldots, \hat{n}_{N}\right\}$ specify a "fiducial" POVM; the completeness property of a POVM implies that these vectors specify a POVM if and only if

$$
0=\sum_{j=1}^{n} \hat{n}_{j}
$$

Any other set, $\left\{R \hat{n}_{j}\right\}$, where $R$ is a three-dimensional rotation, is also a POVM. The frame condition is that

$$
1=\sum_{j=1}^{N} F\left(R \hat{n}_{j}\right)=\sum_{l, m} c_{l m} \sum_{j=1}^{N} Y_{l m}\left(R \hat{n}_{j}\right)=\sum_{l=0}^{\infty} \sum_{m, r=-l}^{l} c_{l m} \mathcal{D}_{m r}^{(l) *}(R) \sum_{j=1}^{N} Y_{l r}\left(\hat{n}_{j}\right)
$$

for all rotations $R$. In writing the last equality, we use

$$
Y_{l m}(R \hat{n})=\sum_{r=-l}^{l} Y_{l r}(\hat{n}) \mathcal{D}_{r m}^{(l)}\left(R^{-1}\right)=\sum_{r=-l}^{l} Y_{l r}(\hat{n}) \mathcal{D}_{m r}^{(l) *}(R),
$$

where $\mathcal{D}_{m r}^{(l)}(R)$ is the irreducible (unitary) matrix representation of the rotation $R$ in the angular-momentum subspace with angular momentum $l$.

We now use the fundamental orthogonality property of the irreducible representations of the rotation group [13]:

$$
\int d \mu_{R} \mathcal{D}_{m r}^{(l) *}(R) \mathcal{D}_{m^{\prime} r^{\prime}}^{\left(l^{\prime}\right)}(R)=\frac{1}{2 l+1} \delta_{l l^{\prime}} \delta_{m m^{\prime}} \delta_{r r^{\prime}}
$$

Here the integration is over the invariant measure $d \mu_{R}$ of the rotation group. Noting that $\mathcal{D}_{00}^{(0)}(R)=1$, we can use this orthogonality relation to invert Eq. (4.7), obtaining the condition $c_{l m} \sum_{j=1}^{N} Y_{l r}\left(\hat{n}_{j}\right)=\delta_{l 0}$ for all $l, m$, and $r$. For $l=0$ this is a trivial normalization constraint, satisfied by choosing $c_{00}=\sqrt{4 \pi} / N$. For $l \geq 1$, we can write the condition in a more illuminating, equivalent form,

$$
c_{l m}=0 \text { for } m=-l, \ldots, l
$$

$$
\begin{array}{ll}
l \geq 1: & \text { or } \\
& 0=\sum_{j=1}^{N} Y_{l r}\left(\hat{n}_{j}\right) \text { for } r=-l, \ldots, l .
\end{array}
$$

These are necessary and sufficient conditions for a frame function $F(\hat{n})$.

The frame conditions (4.10) and (4.11) are a potent restriction. They say that if the $l$ th harmonic is allowed in $F(\hat{n})$, then the unit vectors for the fiducial POVM must satisfy the sum condition (4.11). The choice of fiducial POVM being arbitrary, the sum condition must be satisfied by the unit vectors for all POVMS in the restricted set under consideration. This extension from a fiducial POVM to all POVMs is, however, automatic: If a fiducial POVM satisfies the sum condition (4.11), then the rotation property (4.8) of spherical harmonics guarantees that the condition is satisfied by all POVMs in the restricted set.

The sum condition (4.11) is automatically satisfied for $l=1$ by virtue of the completeness condition (4.6). For higher $l$, if one finds a nonzero value of $\sum_{j=1}^{N} Y_{l r}\left(\hat{n}_{j}\right)$ for just one value of $r$ and just one set of POVM vectors $\left\{\hat{n}_{j}\right\}$, then the $l$ th harmonic must be absent from frame functions. On the other hand, if the sum condition (4.11) is satisfied for a fiducial POVM, then $F(\hat{n})$ can contain the $l$ th harmonic. The expansion coefficients $c_{l m}$ cannot be chosen arbitrarily, of course, since $F(\hat{n})$ must be real and nonnegative. Making $F(\hat{n})$ real is trivial-simply choose $c_{l,-m}=(-1)^{m} c_{l m}^{*}$ - but delineating the region of coefficients that gives rise to nonnegative functions is generally quite 
a difficult task. Nonetheless, we can conclude that the $l$ th harmonic is allowed whenever the sum condition is met, for the following reason. Because the spherical harmonics are bounded functions, sufficiently small expansion coefficients $c_{l m}$ can be combined with $c_{00}=\sqrt{4 \pi} / N$ without making $F$ negative.

The spherical harmonic $Y_{l r}(\hat{n})$ can be regarded as the $r$ th component of a rank- $l$ spherical tensor formed from $\hat{n}$; it is a linear combination of the components of the rank- $l$ symmetric trace-free Cartesian tensor formed from $\hat{n}$. The vanishing of $\sum_{j=1}^{N} Y_{l r}\left(\hat{n}_{j}\right)$ simply says that the sum of these tensors over all the unit vectors in a POVM vanishes. Harmonics $l=1$ and $l=2$ illustrate what is going on. The $l=1$ spherical-tensor components $Y_{1 r}(\hat{n})$ are linear combinations of the Cartesian components of $\hat{n}$ and thus always sum to zero over the unit vectors in a POVM as a consequence of the constraint (4.6). The $l=2$ spherical-tensor components $Y_{2 r}(\hat{n})$ are linear combinations of Cartesian components of the symmetric trace-free two-tensor $n_{k} n_{l}-\frac{1}{3} \delta_{k l}$. Thus $\sum_{j=1}^{N} Y_{2 r}\left(\hat{n}_{j}\right)=0$ if and only if

$$
\sum_{j=1}^{N}\left(n_{j}\right)_{k}\left(n_{j}\right)_{l}=\frac{N}{3} \delta_{k l}
$$

i.e., the sum of the projectors onto the 3 -vectors $\hat{n}_{j}$ is proportional to the 3-dimensional identity operator.

We can make another general statement. If the allowed POVMs are made up of pairs of (subnormalized) orthogonal projectors - i.e., $-\hat{n}$ is in the set of unit vectors if $\hat{n}$ is - then the parity property of the spherical harmonics implies that all odd harmonics are allowed in the frame function. This, of course, is the reason that Gleason's theorem does not hold for qubits if the allowed measurements are restricted to orthogonal projectors.

We turn now to applying the sum condition (4.11) to particular sets of POVMs [14].

\section{B. Restricted sets of POVMs}

\section{Trine measurements}

Consider first the trine measurements, three-outcome measurements described by three unit vectors equally spaced in a plane, which thereby sum to zero. We consider the sum $\sum_{j=1}^{3} Y_{l l}\left(\hat{n}_{j}\right)$ for the particular trine

$$
\hat{n}_{1}=\hat{e}_{x}, \quad \hat{n}_{2,3}=-\frac{1}{2} \hat{e}_{x} \pm \frac{\sqrt{3}}{2} \hat{e}_{z} .
$$

Using $Y_{l l}(\hat{n}) \propto\left(n_{x}+i n_{y}\right)^{l}$, we find that the sum is proportional to $1+(-1)^{l} / 2^{l-1}$. This being nonzero for all $l$ except $l=1$, we conclude that a frame function has no harmonics higher than $l=1$. To establish the precise form of the quantum rule, we must require that the frame function be real and nonnegative. Having ruled out all but harmonics $l=0,1$, we can write the general form as $F(\hat{n})=(1+\hat{n} \cdot \vec{P}) / N$, where $\vec{P}$ is a 3 -vector required to be real by the reality of $F(\hat{n})$. The nonnegativity of $F(\hat{n})$ then requires that $\|\vec{P}\| \leq 1$, leaving us with the standard quantum rule.

\section{Tetrahedral measurements}

Now consider the tetrahedral measurements, four-outcome measurements whose unit vectors point to the vertices of a tetrahedron. Tetrahedral measurements are important as the two-dimensional example of a symmetric informationally complete POVM, i.e., a POVM made up of $d^{2}$ rank-one effects whose pairwise inner products are all the same. The statistics of an informationally complete POVM determine the density operator, and the restriction on the pairwise inner products gives symmetric informationally complete POVMS particularly appealing symmetry properties.

Since the vertices of a tetrahedron satisfy

$$
\sum_{j=1}^{4}\left(n_{j}\right)_{k}\left(n_{j}\right)_{l}=\frac{4}{3} \delta_{k l}
$$

we can conclude immediately, as discussed above, that $\sum_{j=1}^{4} Y_{2 r}\left(\hat{n}_{j}\right)=0$ for all $r$ and all tetrahedra. This means that a frame function for a tetrahedral measurement can contain $l=2$ harmonics and thus does not necessarily follow from the quantum probability rule. 
To investigate the possibility of higher harmonics, consider the particular tetrahedron

$$
\hat{n}_{1}=\hat{e}_{x}, \quad \hat{n}_{2}=-\frac{1}{3} \hat{e}_{x}-\frac{2 \sqrt{2}}{3} \hat{e}_{z}, \quad \hat{n}_{3,4}=-\frac{1}{3} \hat{e}_{x}+\frac{\sqrt{2}}{3} \hat{e}_{z} \pm \sqrt{\frac{2}{3}} \hat{e}_{y} .
$$

For this tetrahedron the $\operatorname{sum} \sum_{j=1}^{4} Y_{l l}\left(\hat{n}_{j}\right)$ is proportional to

$$
1+\left(-\frac{1}{3}\right)^{l}+\left(-\frac{1}{3}+i \sqrt{\frac{2}{3}}\right)^{l}+\left(-\frac{1}{3}-i \sqrt{\frac{2}{3}}\right)^{l}=1+\frac{(-1)^{l}+2(\sqrt{7})^{l} \cos l \alpha}{3^{l}},
$$

where $e^{i \alpha}=-1 / \sqrt{7}+i \sqrt{6 / 7}$. It is not hard to verify that this quantity is nonzero for all $l \neq 1,2,5(\cos 2 \alpha=-5 / 7$ and $\cos 5 \alpha=-121 / 49 \sqrt{7}$ ), implying that a frame function cannot have any harmonics other than $l=0,1,2,5$.

To show that a frame function can have $l=5$ harmonics, rotate the tetrahedron (4.15) by $-90^{\circ}$ about the $y$ axis, obtaining

$$
\hat{n}_{1}=\hat{e}_{z}, \quad \hat{n}_{2}=-\frac{1}{3} \hat{e}_{z}+\frac{2 \sqrt{2}}{3} \hat{e}_{x}, \quad \hat{n}_{3,4}=-\frac{1}{3} \hat{e}_{z}-\frac{\sqrt{2}}{3} \hat{e}_{x} \pm \sqrt{\frac{2}{3}} \hat{e}_{y} .
$$

It is easy to see that for this tetrahedron, $\sum_{j=1}^{4} Y_{l r}\left(\hat{n}_{j}\right)$ vanishes when $r$ is not a multiple of 3 . For $r=0$ this sum is proportional to $\sum_{j=1}^{4} P_{l}\left(\left(n_{j}\right)_{z}\right)=1+3 P_{l}(-1 / 3)$, and for $r=3 n \neq 0$, it is proportional to $\sum_{j=2}^{4} P_{l}^{3 n}\left(\left(n_{j}\right)_{z}\right)=$ $3 P_{l}^{3 n}(-1 / 3)$. For $l=5$, it is easy to check that $P_{5}(-1 / 3)=-1 / 3$ and $P_{5}^{3}(-1 / 3)=0$, so the sum condition (4.11) holds for $l=5$, and a frame function can contain $l=5$ harmonics.

\section{3. $\quad$ Other Measurements}

We now apply our technique to other measurements with nice symmetry properties: the five platonic solids, planar polygons of any degree, and the uniform POVM.

Easiest is the case of the uniform POVM, i.e., a measurement whose outcomes include every direction on the Bloch sphere. There being only one POVM in the restricted set, the frame conditions reduce to the requirement that $F(\hat{n})$ be a real, nonnegative function that integrates to unity on the sphere. In terms of a spherical harmonic expansion, this fixes $c_{00}=1 / \sqrt{4 \pi}$, while the other coefficients are only restricted so as to provide real, nonnegative function values.

Continuing in reverse order, consider the case when the allowed unit vectors lie in a plane, forming a regular polygon with $N$ vertices. For a POVM with unit vectors lying in the $x-y$ plane, it is easy to see that $\sum_{j=1}^{N} Y_{l r}\left(\hat{n}_{j}\right)$ is zero unless $r$ is a multiple of $N$; when $r=n N$, the sum is proportional to $P_{l}^{n N}(0)$, which is zero (nonzero) if $l+n N$ is odd (even). As a consequence, the allowed harmonics for $N$ even are $l=0$ and all odd harmonics, and the allowed harmonics for $N$ odd are $l=0$ and the odd harmonics such that $l \leq N-2$. Notice that the trine is the only regular polygon that gives the quantum probability rule.

The vertices of each of the five platonic solids yield a symmetric set of unit vectors for constructing restricted sets of POVMs. For all five, the projectors onto the unit vectors sum to a multiple of the 3-dimensional identity operator, implying that $l=2$ harmonics are allowed; moreover, for all but the tetrahedron, the unit vectors come in antipodal pairs, meaning that all odd harmonics are allowed. For the octahedron, by considering the $\operatorname{sum} \sum_{j} Y_{l l}(\hat{n})$ over the six unit vectors that point along the $x$ and $-x$ axes and into the four quadrants in the $y-z$ plane, one finds that all even harmonics except $l=0,2$ are ruled out. Similarly, for the cube, this same sum over the tetrahedron (4.15) and its antipodal points rules out all even harmonics except $l=0,2$. For the dodecahedron and icosahedron, additional even harmonics are allowed, and numerical investigation using Mathematica shows these to be $l=4,8,14$. The results for the platonic solids are summarized in Table IVB3

\section{CONCLUSION}

Gleason's theorem reveals that from this "measurement first" point of view, the quantum probability rule is implicit in the structure of measurements themselves. Use of generalized measurements greatly simplifies the proof of Gleason's theorem, extends its applicability, and reduces the conceptual overhead of quantum theory. Attention is thereby refocused to the tougher task of justifying the form of quantum measurements $[3,[4,[5]$. 


\begin{tabular}{|l|r|}
\hline Platonic solid & Allowed harmonics \\
\hline tetrahedron & $0,1,2,5$ \\
octahedron & $0,2, \&$ odds \\
cube & $0,2, \&$ odds \\
dodecahedron & $0,2,4,8,14, \&$ odds \\
icosahedron & $0,2,4,8,14, \&$ odds \\
\hline
\end{tabular}

Investigating particular measurements and their allowable probability distributions complements the investigation of Kochen-Specker colorable sets 15]. Considering particular sets of POVMS allows us to explore the range of probability distributions that lie between the quantum probability rule and Kochen-Specker truth assignments.

This work naturally leads to the question of whether POVMs ought to be considered, in some sense, more fundamental than standard projection-valued measures. The reason for thinking so is not just the simplicity of proofs. Foremost is the notion of "fuzziness" that effects capture, a notion essential for practical purposes. Secondly, in thinking of quantum mechanics operationally, nothing singles out projection measurements for fundamental status. While it's true that effects themselves are convex combinations of projection operators, POVMs needn't be convex combinations of projection-valued measures [16]. Finally, POVMs are useful measurements, as demonstrated by their increasing use in the field of quantum information [17]. All these reasons point toward a fundamental role for POVMs.

\section{Acknowledgments}

This work was supported in part by Office of Naval Research Grant No. N00014-00-1-0578.

[1] S. Kochen and E. P. Specker, J. Math. Mech. 17, 59 (1967).

[2] A. M. Gleason, J. Math. Mech. 6, 885 (1957).

[3] C. A. Fuchs, "Quantum Mechanics as Quantum Information (and only a little more)," quant-ph/0205039

[4] R. J. Greechie and D. J. Foulis, Int. J. Th. Phys. 34, 1369 (1995).

[5] G. Ludwig, Foundations of Quantum Mechanics, Springer, Berlin, 1983.

[6] K. Kraus, States, Effects, and Operations: Fundamental Notions of Quantum Theory, Lecture Notes in Physics, Vol. 190, (Springer-Verlag, Berlin, 1983).

[7] P. Busch, M. Grabowski, and P. Lahti, Operational Quantum Physics (Springer, Berlin, 1995), 2nd corrected printing, 1997.

[8] P. Busch, "Resurrection of von Neumann's No-Hidden-Variables Theorem," quant-ph/ 9909073

[9] D. A. Meyer, Phys. Rev. Lett. 83, 3751 (1999).

[10] R. Clifton and A. Kent, Proc. Roy. Soc. Lond. A 456, 2101 (2000).

[11] D. M. Appleby, Phys. Rev. A 65, 022105 (2002).

[12] A. Cabello, Phys. Rev. A 65, 052101 (2002).

[13] W. Tung, Group Theory in Physics (World Scientific, Philadelphia, 1993).

[14] Our approach to proving Gleason-type theorems for restricted sets of qubit POVMs was inspired by Peres's proof of the standard Gleason theorem in Sec. 7-2 of A. Peres, Quantum Theory: Concepts and Methods (Kluwer, Dordrecht, The Netherlands, 1993). The context there is a 3-dimensional real vector space, with measurements described by orthonormal triads of vectors. A frame function is a function $F$ on unit vectors, which sums to unity on any orthonormal triad. Assuming $F$ is continuous, it can be expanded in terms of spherical harmonics, as in Eq. (4.2). The quantum probability rule, $F(\hat{n})=\operatorname{tr}(W|\hat{n}\rangle\langle\hat{n}|)=\langle\hat{n}|W| \hat{n}\rangle=\sum_{j, k} W_{j k} n_{j} n_{k}$, has only $l=0,2$ harmonics. Peres's proof proceeds by showing that the $l$ th harmonic of $F$, i.e., $\sum_{m} c_{l m} Y_{l m}(\hat{n})$, sums to $\delta_{l 0}$ on orthonormal triads, but his proof of this crucial result is flawed because the rotation-group matrix elements $\mathcal{D}_{m r}^{(l) \prime}$ and $\mathcal{D}_{m r}^{(l) \prime \prime}$ in his Eq. (7.18) depend on $\theta$ and $\phi$. The more powerful frame conditions (4.10) and 4.11, however, immediately imply Peres's result and also imply the quantum probability rule. For the triad $\hat{n}_{1}=\hat{e}_{x}, \hat{n}_{2}=\left(\hat{e}_{y}-e_{z}\right) / \sqrt{2}, \hat{n}_{3}=\left(\hat{e}_{y}+e_{z}\right) / \sqrt{2}$, the sum $\sum_{j=1}^{3} Y_{l l}\left(\hat{n}_{j}\right)$ is proportional to $1+i^{l} / 2^{l / 2-1}$, which is nonzero except for $l=2$, thus ruling out all harmonics except $l=0,2$. As discussed in the text, $\sum_{j=1}^{3} Y_{2 r}\left(\hat{n}_{j}\right)=0$ for all $r$ and all orthonormal triads because the projectors onto the vectors in an orthonormal triad sum to the 3-dimensional identity. We thus have that the frame function can be written as $F(\hat{n})=\operatorname{tr}(W|\hat{n}\rangle\langle\hat{n}|)$ for some Hermitian operator $W$. Nonnegativity and normalization of the frame function imply that $W$ is positive with unit trace.

[15] A. Cabello, "Kochen-Specker Theorem for a Single Qubit," quant-ph/0210082

[16] G. M. D'Ariano and P. L. Presti, "Classical and Quantum Noise in Measurements and Transformations," quant-ph/0301110 
[17] M. A. Nielsen and I. L. Chuang, Quantum Computation and Quantum Information, (Cambridge University Press, Cambridge, 2000). 